\title{
ALTERATIONS IN REDOX HOMEOSTASIS FOLLOWING REPEATED SPRINT TRAINING
}

\author{
Domagoj Marijančević1, Nada Vrkić², Igor Jukićc ${ }^{3}$ and Daniel Bok ${ }^{3}$ \\ ${ }^{1}$ Laboratory of Endocrinology, Department of Oncology and Nuclear Medicine, \\ Sestre Milosrdnice University Hospital Center, Zagreb, Croatia \\ ${ }^{2}$ Faculty of Pharmacy and Biochemistry, University of Zagreb, Zagreb, Croatia; Department \\ of Clinical Chemistry, Sestre Milosrdnice University Hospital Center, Zagreb, Croatia \\ ${ }^{3}$ Faculty of Kinesiology, University of Zagreb, Zagreb, Croatia
}

Original scientific paper

DOI: $10.26582 / \mathrm{k} .52 .1 .3$

\begin{abstract}
:
This study examined the effects of a 6 -week repeated sprint training on redox-based homeostasis and their association with muscle damage. Fifteen male physical education students (aged $20.0 \pm 1.0$ years; body weight $77.7 \pm 6.0 \mathrm{~kg}$; height $181.0 \pm 4.4 \mathrm{~cm}$; \%body fat $8.7 \pm 3.0 \%$ ), familiar with intermittent activities, volunteered to participate in the study. Experimental training program consisted of 2-3 sets of 6-10 straight-line or shuttle 20 -m repeated sprints with departures every 25 seconds and a 2 -minute inter-set passive recovery. The training intervention lasted six weeks during which 18 training sessions were performed. The levels were measured of the following: $15-\mathrm{F}_{2 \mathrm{t}}$-isoprostanes in plasma and 24-hour urine; superoxide dismutase, glutathione peroxidase and glutathione reductase in erythrocytes; uric acid and creatine kinase in serum after the first and the penultimate training session. The level of muscle damage following the repeated sprint exercise was not significantly altered (402 to $496 \mathrm{U} / \mathrm{L} ; \mathrm{p}=.151)$ and had no significant associations with the changes in markers depicting redox-homeostasis. A significant increase in plasma $15-\mathrm{F}_{2 \mathrm{t}}$-isoprostanes $(0.32$ to $0.56 \mathrm{ng} / \mathrm{mL} ; \mathrm{p}=.026$ ), and a subsequent decrease in glutathione reductase ( 7.7 to $3.4 \mathrm{U} / \mathrm{g} \mathrm{Hb} ; \mathrm{p}<.001$ ) were observed. Urinary $15-\mathrm{F}_{2 \mathrm{t}}$-isoprostane levels were $25 \%$ greater at post-training, although this increase did not reach statistical significance. These results indicate that repeated sprint training stimulates the equilibrium in redox homeostasis developing antioxidant protection to the constantly increasing training load.
\end{abstract}

Key words: training program, muscle damage, oxidative stress, $15-F_{2 t}$-isoprostanes, antioxidant status, high-intensity interval training

\section{Introduction}

Regular physical exercise is recognized as an important contributor to health (Garber, et al., 2011). However, acute response to exercise results in the increased production of reactive oxygen species (ROS), which may lead to oxidative stress (OS) (Finaud, Lac, \& Filaire, 2006). An excessive ROS production can cause cellular damage and chronic inflammation, which is associated with the development of various diseases (de Sousa, et al., 2017), and accentuate aging process (Bouzid, Filaire, McCall, \& Fabre, 2015). Besides being responsible for cellular damage, ROS are also important molecules that serve as secondary messengers in skeletal muscle signaling cascades, so their role is essential for muscle adaptation after training (Lewis, Howatson, Morton, Hill, \& Pedlar, 2015). The production of ROS is dependent on exercise intensity and volume (Knez, Jenkins, \& Coombes,
2014) and it can vary considerably depending on the athletes' fitness level, training phase and environmental conditions (Lewis, et al., 2015). However, negative post-exercise effects of acute OS can be counteracted to a certain degree by the acute increase of antioxidants such as superoxide dismutase (SOD), glutathione peroxidase (GPx) and glutathione reductase (GR) (Finaud, et al., 2006). These acute reactions to exercise seem to cause a decrease in pro-oxidants and an increase in antioxidants after periods of physical training regardless of intensity, volume and type of exercise (de Sousa, et al., 2017). Generally, exercise training of any kind is recommended to all populations because it may protect them from OS by reinforcing their antioxidant defenses.

Repeated sprint training (RST) is a form of a high-intensity interval training (HIIT) in which short-duration all-out sprints are interspersed with 
short recovery periods (Buchheit \& Laursen, 2013a). This type of training is often used by team sports players since, during a game, they are required to perform repeated bursts of intensive activities interspersed with very short recovery periods (Bishop, Girard, \& Mendez-Villanueva, 2011). Indeed, RST has shown to be effective in improving power, speed, repeated sprint ability and endurance, which may transfer positively on players' game performance (Taylor, Macpherson, Spears, \& Weston, 2015). Besides being used to improve athletes' fitness, HIIT formats have recently become a very popular and time-efficient training strategy for health improvement among general population (Gibala, 2007). Studies show that HIIT is very effective in improving cardiorespiratory fitness in sedentary population (Weston, Taylor, Batterham, \& Hopkins, 2014) and cardiometabolic health in normal and overweight/obese people (Batacan, Duncan, Dalbo, Tucker, \& Fenning, 2017) as well as in patients with various chronic cardiometabolic diseases (Weston, Wisløff, \& Coombes, 2014). Although HIIT has been recognized and accepted as an effective training modality for fitness and health improvement, there is very little information about the effects of RST, a specific HIIT format, on health in general and, especially, on alterations in redox homeostasis (ARH). Namely, a great anaerobic demand, stemming from the repeated all-out sprints, and increasing aerobic contribution throughout a bout, as the sprints are done with very little recovery in between, may increase the production of ROS and cause OS. Previous studies have reported conflicting results in which an increase (Clifford, et al., 2016; Escobar, et al., 2009; Jówko, Długołęcka, Makaruk, \& Cieśliński, 2015; Peart, et al., 2013), no change (Bloomer, et al., 2006; Clifford, et al., 2016; Farney, et al., 2012; Leeder, et al., 2014) and even a decrease (Goods, et al., 2016) in OS markers were observed following an acute repeated sprint (RS) exercise. Similarly, an increase (Escobar, et al., 2009; Jówko, et al., 2015), no change (Peart, et al., 2013) and a decrease (Farney, et al., 2012) in parameters of antioxidant capacity/activity were observed after acute RS exercises. These discrepancies in acute responses can be attributed to different modes and protocols of RS exercises used in the studies, to the differences in study participants' fitness levels, as well as in ARH markers used for analysis and in measurement time-points. Such opposing acute responses render the prediction of chronic adaptation to RST almost impossible. Namely, in a single study investigating training effects of a cycling RST on antioxidant activity Hellsten, Apple, and Sjödin (1996) reported that six weeks of RST, consisting of three training sessions per week, did not lead to an increase of muscle antioxidant enzymes, while a much more intensive one week training period, consisting of two training sessions per day for seven consecutive days, did lead to a significant increase in muscle GPx and GR. These findings suggest that the level of antioxidant protection in the muscles is related to training volume. As most training programs used in practice follow undulating and progressive increase in training volume to induce adaptation-optimal stress, it is important to investigate whether these constant increases in volume could create positive ARH and provide protection from more intensive RS exercises that are utilized at the end of the training program. Additionally, since Hellsten et al. (1996) used cycling repeated sprints and only measured antioxidant activity, there is still a lack of information about the effects of running RST protocols on both the OS and antioxidant activity. As opposed to cycling, which is an activity comprised of concentric muscle actions only, running, and especially sprinting, is an activity in which high-intensity eccentric muscle contractions are represented. As eccentric contractions are mostly responsible for inducing muscle damage (Douglas, Pearson, Ross, \& McGuigan, 2016) and the muscle damage is reported to be one of the important factors contributing to the increase of OS (Nikolaidis, et al., 2008), it is important to investigate the effects of running RS exercise on ARH.

Therefore, the main aim of the study was to investigate the effects of a running RST on the markers of OS and antioxidant activity. Additionally, the study investigated whether muscle damage serves as a contributing factor in ARH after a training period. It was hypothesized that a six-week RST would result in the reduction of OS and in the increment of antioxidant activity, and that these alterations would be influenced by a reduced post-training muscle damage.

\section{Methods}

\section{Participants}

Fifteen male physical education students volunteered to participate in the study (age 20.0 1.0 years; body weight $77.7 \pm 6.0 \mathrm{~kg}$; body height $181.0 \pm 4.4 \mathrm{~cm}$; $\%$ body fat $8.7 \pm 3.0 \%$ ). A statistical power analysis was performed for sample size estimation, based on the data from a published study (Margonis, et al., 2007). With a power of 0.90 and a two-tailed level set at 0.05 , the minimum number of participants required to detect a 6.4-fold increase of the $15-\mathrm{F}_{2 \mathrm{t}}$-isoprostanes $\left(15-\mathrm{F}_{2 \mathrm{t}}-\mathrm{IsoP}\right)$ concentration $(\mathrm{SD}=10 \mathrm{ng} / \mathrm{mL})$ was estimated as twelve. $15-\mathrm{F}_{2 \mathrm{t}}$-IsoP has been selected because it is widely regarded as the gold standard marker for the assessment of oxidative perturbations in the redox biology of exercise. Participants were recruited for the study through oral announcements of the study, which were delivered on several occasions at the begin- 
ning of their university classes. All participants were screened for cardiovascular diseases, muscle injuries or ongoing medical treatment with the Physical Activity Readiness Questionnaire (PAR-Q) before their inclusion into the experimental protocol. The basic inclusion criterion was nonparticipation in any organized physical activity program or sport for three months before and throughout the study. Participants were, however, allowed to be physically active and to exercise during practical classes they were obligated to undertake as their course requirement. As they were physical education students, all participants were either former athletes or had participated in extensive training of a certain sport, so they were familiar with intermittent activities. Participants were instructed to refrain from alcohol and cigarette as well as antioxidant supplementation consumption throughout the study. Before the study, the experimental procedures and potential risks were explained in detail to the participants and they all provided written informed consent. The study was designed in accordance with the Declaration of Helsinki and was approved by the Ethical Committee of the Faculty of Pharmacy and Biochemistry, University of Zagreb.

\section{Study design}

The experimental protocol was carried out within nine weeks. Anthropometric measurements were performed during the first and the final week of the experimental protocol along with the other fitness tests which were required as this study was conducted as part of a larger sports physiology study. In order to familiarize the participants with the training program, two 60 -minute familiarization training sessions were scheduled during the second week. The training intervention started on the third week and lasted for six weeks during which 18 training sessions were performed. Training sessions were scheduled on the same time of the day (from 4 to $6 \mathrm{pm}$ ), three times a week, with a 48-hour gap between the sessions. All training sessions were performed in a handball-court gym on a wooden floor and were supervised by a strength and conditioning coach. In order to test the protective effects of undulating increase in training load on OS and antioxidant activity, blood samples were taken after the first intervention training session (pre-training time point), which was least intensive, and the penultimate training session (posttraining time point), which was the most intensive one. Blood samples were taken within 20 minutes after the training sessions. This protocol is justified by a relatively short elimination half-life of $15-\mathrm{F}_{2 \mathrm{t}}$-IsoP in blood. Judging from the studies that measured $15-\mathrm{F}_{2 \mathrm{t}}$-IsoP immediately after exercise, it appears that the plasma $15-\mathrm{F}_{2 \mathrm{t}}-\mathrm{IsoP}$ levels return to baseline values in less than one hour after exercise cessation.

\section{Training intervention}

Each training session started with the standardized warm-up consisting of low-intensity running, dynamic stretching and progressive short-distance accelerations for the total duration of 18 minutes. The main part of the session consisted of 2-3 sets of 6-10 straight-line or shuttle 20-meter repeated sprints with departures every 25 seconds and a 2-minute inter-set passive recovery (Table 1). Recovery between sprints was active as the participants were required to jog back to the starting line and assume starting position three seconds before the audio signal for the next sprint. The entire training protocol for each session was prerecorded as an audio file and was then played on a personal computer, so that timing for the commencement of each sprint and duration of recovery interval could be fully controlled. After the starting signal, the participants were required to run the $20-\mathrm{m}$ distance as fast and possible. As this study was conducted as part of a larger study in which two experimental groups were formed, 12 participants performed straight-line repeated sprints while the other three participants performed shuttle repeated sprints in all training sessions. The total training volume in both groups was equal, so this difference between the two experimental protocols should not be reflected in the results of this particular study; therefore, we decided to conduct the analysis on pooled data. In order to comply with the progression and undulation principle of the training, the number of sets and repetitions was progressively increased throughout the six-week intervention period. During the intervention period, each partici-

Table 1. Experimental training program

\begin{tabular}{|c|c|c|c|c|c|c|}
\hline & Week 1 & Week 2 & Week 3 & Week 4 & Week 5 & Week 6 \\
\hline Monday & $\begin{array}{c}2 \times 6 \times 20 \mathrm{~m} \\
12 \text { sprints }\end{array}$ & $\begin{array}{c}3 \times 6 \times 20 \mathrm{~m} \\
18 \text { sprints }\end{array}$ & $\begin{array}{c}2 \times 8 \times 20 \mathrm{~m} \\
16 \text { sprints }\end{array}$ & $\begin{array}{c}3 \times 8 \times 20 \mathrm{~m} \\
24 \text { sprints }\end{array}$ & $\begin{array}{c}2 \times 10 \times 20 \mathrm{~m} \\
20 \text { sprints }\end{array}$ & $\begin{array}{c}3 \times 10 \times 20 \mathrm{~m} \\
30 \text { sprints }\end{array}$ \\
\hline Wednesday & $\begin{array}{c}2 \times 6 \times 20 \mathrm{~m} \\
12 \text { sprints }\end{array}$ & $\begin{array}{c}3 \times 6 \times 20 \mathrm{~m} \\
18 \text { sprints }\end{array}$ & $\begin{array}{c}2 \times 8 \times 20 \mathrm{~m} \\
16 \text { sprints }\end{array}$ & $\begin{array}{c}3 \times 8 \times 20 \mathrm{~m} \\
24 \text { sprints }\end{array}$ & $\begin{array}{c}2 \times 10 \times 20 \mathrm{~m} \\
20 \text { sprints }\end{array}$ & $\begin{array}{c}3 \times 10 \times 20 \mathrm{~m} \\
30 \text { sprints }\end{array}$ \\
\hline Friday & $\begin{array}{c}2 \times 6 \times 20 \mathrm{~m} \\
12 \text { sprints }\end{array}$ & $\begin{array}{c}3 \times 6 \times 20 \mathrm{~m} \\
18 \text { sprints }\end{array}$ & $\begin{array}{c}2 \times 6 \times 20 \mathrm{~m} \\
12 \text { sprints }\end{array}$ & $\begin{array}{c}3 \times 8 \times 20 \mathrm{~m} \\
24 \text { sprints }\end{array}$ & $\begin{array}{c}2 \times 10 \times 20 \mathrm{~m} \\
20 \text { sprints }\end{array}$ & $\begin{array}{c}2 \times 6 \times 20 \mathrm{~m} \\
12 \text { sprints }\end{array}$ \\
\hline $\begin{array}{l}\text { Total weekly } \\
\text { training load }\end{array}$ & $\begin{array}{c}36 \text { sprints } \\
720 \mathrm{~m}\end{array}$ & $\begin{array}{l}54 \text { sprints } \\
1080 \mathrm{~m}\end{array}$ & $\begin{array}{c}44 \text { sprints } \\
880 \mathrm{~m}\end{array}$ & $\begin{array}{l}72 \text { sprints } \\
1440 \mathrm{~m}\end{array}$ & $\begin{array}{l}60 \text { sprints } \\
1200 \mathrm{~m}\end{array}$ & $\begin{array}{c}72 \text { sprints } \\
1440 \mathrm{~m}\end{array}$ \\
\hline
\end{tabular}


pant executed 338 sprints and covered 6,760 meters in total. This training program was chosen as it elicits significant improvements in maximal oxygen uptake, speed, agility, power, and repeated sprint ability (Bok, Jukić, Milanović, \& Gregov, 2015). In order to maintain maximal intensity throughout the entire training session, a constant verbal encouragement was provided by the training supervisor.

\section{Anthropometric measurements}

Anthropometric measurements were performed in the fitness laboratory during the first and the ninth week. Portable anthropometer (Holtain, Instruments Ltd., Crosswell, UK) was used for measuring body height, while segmental body composition analyzer (BC-418, Tanita Corporation, Tokyo, Japan) was used for assessing body weight and body fat percentage. Anthropometric measurements were taken by the same experienced member of the fitness laboratory staff.

\section{Blood and urine sampling}

Blood was collected within 20 minutes postexercise after each experimental phase, at the same time at each stage to control for diurnal variation in analytes concentrations. Phlebotomy was performed after at least 15 minutes of resting in a seated position by the expert phlebotomists according to the national recommendations for venous blood sampling issued by the Croatian Society of Medical Biochemistry and Laboratory Medicine (Nikolac, Šupak-Smolčić, Šimundić, \& Ćelap, 2013). All blood samples were drawn from the antecubital vein, using a $20-\mathrm{G}$ straight needle (BD), directly into $\mathrm{K}_{2}$ EDTA vacuum tubes $(1 \mathrm{x} 2$ $\mathrm{mL}$; BD, Vacutainer, Franklin Lakes, NJ, USA) and serum vacuum tubes with clot activator $(1 \times 10 \mathrm{~mL}$; BD, Vacutainer, Franklin Lakes, NJ, USA). EDTA tubes were immediately centrifuged (at $1370 \mathrm{x}$ g for 10 minutes) to separate erythrocytes from plasma, while serum tubes were left to clot for $45 \mathrm{~min}$ before centrifugation. All biochemistry routine parameters were measured immediately after the serum preparation. Subsequently, erythrocytes were washed three times with a cold isotonic saline solution. Erythrocytes, plasma, and serum supernatant were aspirated into a series of $1 \mathrm{~mL}$ aliquots and stored at $-80^{\circ} \mathrm{C}$ until analysis.

A 24-hour urine sample was collected four days post-exercise, following each experiment phase, using a plastic container $(1 \times 3000 \mathrm{~mL}$; Vacutest Kima, PD, Italy). Participants were previously trained in how to accurately collect a 24-hour urine sample. When the collection was completed, the total urine volume was measured upon arrival in the laboratory. The total 24-hour urine sample was agitated to obtain homogeneous aliquots. One 10
$\mathrm{mL}$ aliquot of each participant's urine sample was stored at $-80^{\circ} \mathrm{C}$ for later analysis.

\section{Biochemical markers of muscle damage and redox-based homeostasis}

Creatine kinase (CK) and uric acid (UA) were determined using AU2700 plus analyzer (Beckman Coulter, Brea, CA, USA), according to the manufacturer's specifications and using proprietary reagents: $\mathrm{N}$-acetylcysteine-activated IFCC UV method (CK) and uricase/peroxidase colorimetric method (UA).

Plasma levels of free $15-\mathrm{F}_{2 \mathrm{t}}$-IsoP were measured using a competitive enzyme-linked immunoassay (ELISA) kit (Oxford Biomedical Research, Inc., Oxford, MI, USA). Solid-phase extraction (SPE) was carried out with Oasis HLB extraction plate $10 \mathrm{mg}, 30 \mu \mathrm{m}$ SPE columns (Waters, Milford, MA, USA). Plasma ( $1 \mathrm{~mL}$; $\mathrm{pH}$ adjusted to 3) was pre-purified on a C18 Sep Pak column and then loaded onto a Silica Sep Pak column. After two wash steps, the bound $15-\mathrm{F}_{2 \mathrm{t}}$-IsoP was eluted from the SPE column with a mixture of ethyl acetate/heptane and evaporated to dryness. Before performing ELISA, samples were reconstituted in $1 \mathrm{~mL}$ of dilution buffer (provided with the kit) and the assay was performed as recommended by the manufacturer. The standard curve ranged between

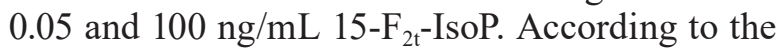
information provided, the antibody cross-reacts weakly with $9 \alpha, 11 \beta$-prostaglandin-F $\alpha \alpha(4.1 \%)$ and 13,14-dihydro-15-keto-F2 $\alpha$ (3\%). Within-laboratory precision $(\% \mathrm{CV}$ intra-assay variation based on the standard curve samples run in triplicates over five days) was less than $20 \%$.

$15-\mathrm{F}_{2 \mathrm{t}}$-IsoP levels in 24-hour urine were quantified using a competitive enzyme-linked immunosorbent assay (ELISA) as described in the manufacturer's protocol (Oxford Biomedical Research, Inc., Oxford, MI, USA). To eliminate interferences due to non-specific binding, a 24-hour urine sample $(1 \mathrm{~mL})$ was diluted 1:4 using enhanced dilution buffer before performing the assay. Oxford Biomedical Research has found that an average of $50 \%$ of the isoprostane excreted in human urine is conjugated to glucuronic acid. The extent of glucuronidation among individuals ranges significantly from $28 \%$ to $80 \%$. In addition, specimens were pretreated with $\beta$-glucuronidase prior to the analysis in order to provide a more accurate assessment of redox-based homeostasis. The assay range, precision and the cross-reactivity of the antibody were the same as described above for the corresponding plasma kit.

Superoxide dismutase, glutathione peroxidase and glutathione reductase activities in erythrocytes were evaluated by the spectrophotometric method using commercially available kits (RANSOD, 
RANSEL, and GLUT RED, respectively; Randox, Crumlin, UK). The antioxidant enzyme activities were measured at $37{ }^{\circ} \mathrm{C}$ and expressed in $\mathrm{U} / \mathrm{g}$ $\mathrm{Hb}$. Hemoglobin was assessed by a standard cyanmethemoglobin method, on ADVIA 2120i hematology analyzer (Siemens Healthcare Diagnostics, Eschborn, Germany). Within-laboratory precision (\% CV intra-assay variation based on the control samples run in triplicates over five days) for SOD, GPx, and GR were 6.5, 5.8, and 5.4\%, respectively.

\section{Statistical analysis}

Normality of data distribution was assessed using the Shapiro-Wilk test. Statistical significance of the pre- to post-differences were tested by Wilcoxon matched pairs test and the data were presented as the median and interquartile range (IQR). Relations between data were analyzed by Spearman correlation analysis. Level of statistical significance was set at $\mathrm{p}<.05$. Statistical analysis was performed using MedCalc Statistical Software version 19.1 (MedCalc Software bv, Ostend, Belgium; https://www.medcalc.org; 2019).

\section{Results}

The RST program did not affect the marker of muscle damage since no significant post-training difference was determined in CK activities (Table 2).

The responses of the redox-based homeostasis markers induced by the RST program are presented in Table 2. The statistically significant changes were observed in repeated measures of plasma $15-\mathrm{F}_{2 \mathrm{t}}$-IsoP concentration and GR activity. While the median plasma $15-\mathrm{F}_{2 \mathrm{t}}$-IsoP increased by $75 \%$ (from 0.32 to $0.56 \mathrm{ng} / \mathrm{mL} ; \mathrm{p}=.026$ ), the median GR decreased by $56 \%$ (from 7.7 to $3.4 \mathrm{U} / \mathrm{g} \mathrm{Hb} ; \mathrm{p}<.001$; Figure 1).

In 24-hour urine samples, $15-\mathrm{F}_{2 \mathrm{t}}$-IsoP levels were $25 \%$ greater in post-training, although these increases did not reach statistical significance. In addition, following the RST, erythrocyte SOD and GPx activities were $11 \%$ and $27 \%$ lower, although these decreases were not statistically significant. Serum UA concentration remained close to the baseline values throughout the training program, showing no time effects (Table 2).

Table 2. Changes in the biochemical markers of muscle damage and redox-based homeostasis induced by the repeated sprint training program

\begin{tabular}{lccc}
\hline Biochemical marker (unit) & Pre & Post & $\mathrm{p}$ \\
\hline $\mathrm{CK}(\mathrm{U} / \mathrm{L})$ & $402(288$ to 579$)$ & $496(414$ to 812$)$ & .151 \\
Plasma $15-\mathrm{F}_{2 \mathrm{t}}$-lsoP $(\mathrm{ng} / \mathrm{mL})$ & $0.32(0.13$ to 0.57$)$ & $0.56(0.30$ to 1.24$)$ & $.026^{*}$ \\
Urinary $15-\mathrm{F}_{2 \mathrm{t}}$-lsoP $(\mathrm{ng} / 24 \mathrm{~h})$ & $1.02(0.77$ to 1.72$)$ & $1.31(0.91$ to 1.99$)$ & .121 \\
SOD $(\mathrm{U} / \mathrm{g} \mathrm{Hb})$ & $372.3(300.8$ to 431.4$)$ & $354.2(310.2$ to 392.0$)$ & .489 \\
GPx $(\mathrm{U} / \mathrm{g} \mathrm{Hb})$ & $16.6(12.5$ to 28.1$)$ & $13.2(11.9$ to 17.0$)$ & .119 \\
GR $(\mathrm{U} / \mathrm{g} \mathrm{Hb})$ & $7.7(5.0$ to 9.0$)$ & $3.4(3.3$ to 3.8$)$ & $<.001^{*}$ \\
Uric acid $(\mu \mathrm{mol} / \mathrm{L})$ & $300(275$ to 338$)$ & $325(290$ to 347$)$ & .229 \\
\hline
\end{tabular}

Note. Values are presented as median (interquartile range). Abbreviations: Pre - time point after the first training session; Post time point after the penultimate repeated sprint training; $\mathrm{CK}$ - creatine kinase; $15-\mathrm{F}_{2 \mathrm{t}}-\mathrm{IsoP}-15-\mathrm{F}_{2 \mathrm{t}}$-isoprostane; SOD - superoxide dismutase; GPx - glutathione peroxidase; GR - glutathione reductase; * - significant difference in comparison to the Pre value $(p<.05)$.
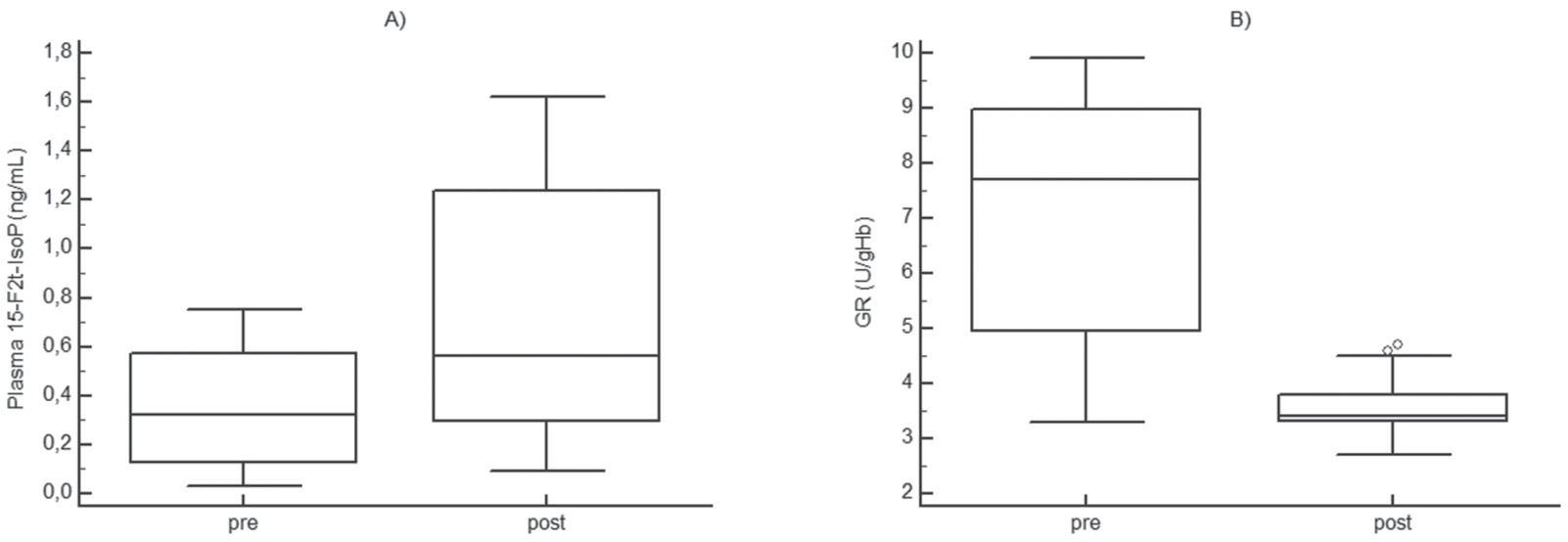

Figure 1. Statistically significant pre- to post-differences in ARH markers caused by RST; A) plasma 15- $F_{2 t}-I s o P(n g / m L)$ and B) $G R(U / g ~ H b)$. Values are presented as median (interquartile ranges). Abbreviations: GR - glutathione reductase; Pre - time point after the first training session; Post - time point after the penultimate repeated sprint training. 
Table 3. Spearman's rank correlation between biochemical markers of muscle damage and redox-based homeostasis induced by the repeated sprint training program

\begin{tabular}{lcc}
\hline & $\begin{array}{c}\text { Correlation coefficient }(r) \\
(95 \% \text { Confidence Interval for } r)\end{array}$ & $p$ \\
\hline$\Delta$ CK vs. $\Delta$ plasma $15-F_{2 t}$-IsoP & $0.35(-0.20$ to 0.73$)$ & .200 \\
$\Delta$ CK vs. $\Delta$ urinary $15-\mathrm{F}_{2 t}$-IsoP & $0.04(-0.48$ to 0.54$)$ & .889 \\
$\Delta$ CK vs. $\Delta$ SOD & $0.07(-0.46$ to 0.56$)$ & .800 \\
$\Delta$ CK vs. $\Delta$ GPx & $0.14(-0.40$ to 0.61$)$ & .612 \\
$\Delta$ CK vs. $\Delta$ GR & $-0.09(-0.58$ to 0.44$)$ & .752 \\
$\Delta$ CK vs. $\Delta$ Uric acid & $0.10(-0.44$ to 0.58$)$ & .723 \\
\hline
\end{tabular}

Note. Abbreviations: $\Delta$ - the mean difference (\%) between measurements at different time points; $15-F_{2 t}-$ IsoP $-15-F_{2 t}$-isoprostane; SOD - superoxide dismutase; GPx - glutathione peroxidase; GR - glutathione reductase.

There were no significant correlations between pre-to-post change in CK and pre-to-post change in any marker of redox homeostasis (Table 3).

\section{Discussion and conclusions}

This was the first study to report the effects of a 6-week running RST on ARH and their association with muscle damage. The main findings of the study were that: 1) the level of muscle damage following RS exercise was not significantly altered after the RST program; 2) there was a significant increase in plasma $15-\mathrm{F}_{2 \mathrm{t}}-\mathrm{IsoP}$ and a non-significant increase in the urinary $15-\mathrm{F}_{2 t}-$ IsoP after the RST program; 3) there was a significant decrease in GR, but not in any other marker of antioxidant activity after the RST program; and 4) the pre-topost-training changes in markers depicting ARH established no significant associations with the preto-post changes in CK.

The level of CK obtained at the pre- and posttraining time points suggests that a high muscle damage occurs as a result of the RS exercise. This was consistent with the previously published studies in which similar CK values of $592 \mathrm{U} / \mathrm{L}$ were registered (Clifford, et al., 2016); however, even much higher values of $776 \mathrm{U} / \mathrm{L}$ (Howatson \& Milak, 2009) and $1120.7 \mathrm{U} / \mathrm{L}$ (Woolley, Jakeman, \& Faulkner, 2014) were also reported after different running $\mathrm{RS}$ exercises. Interestingly, much lower $\mathrm{CK}$ values of $230.6 \mathrm{U} / \mathrm{L}$ (Jówko, et al., 2015) and $166 \mathrm{U} / \mathrm{L}$ (Bloomer, et al., 2006) were reported in studies investigating cycling RS exercises, which was probably caused by a different muscle action utilized in cycling versus running. Lower $\mathrm{CK}$ values obtained in our study were probably due to the less stressful $\mathrm{RS}$ exercise utilized here in comparison to the ones utilized in other running RS studies. Indeed, the RS exercises analyzed in our study were comprised of two sets of six $10 \times 20$-m sprinting and three sets of $10 \times 20$-m sprinting prior to the initial and the final measurement, respectively, while the $40 \times 15$ m (Woolley, et al., 2014), $15 \times 30 \mathrm{~m}$ (Howatson \& Milak, 2009) and $20 \times 30 \mathrm{~m}$ (Clifford, et al., 2016) $\mathrm{RS}$ protocols were used in other studies. Since those
RST protocols were comprised of longer sprint distances ran within a single set (Clifford et al., 2016; Howatson \& Milak, 2009) and even had a greater number of repetitions (Woolley, et al., 2014), a greater metabolic and neuromuscular stress could be expected in those studies (Buchheit \& Laursen, 2013b), which could than induce a greater muscle damage. However, different measurement time points also contributed to dissimilar $\mathrm{CK}$ values as the blood drawing procedure was performed within the first hour post-exercise in this study, while the reported peak CK values in other studies were obtained 24 hours post-exercise. Namely, peak CK values are usually reached 24 hours after highintensity sport activities in which RS sequences are frequently executed (Silva, et al., 2018). Interestingly, 1-hour post-exercise CK values, reported in some of the above-cited studies, were $362 \mathrm{U} / \mathrm{L}$ (Clifford, et al., 2016) and 376 U/L (Woolley, et al., 2014), which was similar to the values obtained in the present study. It can be implicated that the level of muscle damage in our study was even greater, but the measurement time point prevented us from capturing the peak $\mathrm{CK}$ values. Nonetheless, we can conclude that the level of muscle damage was very high as such CK values were actually compatible to the ones registered after a competitive soccer match (Bok \& Jukić, 2019). Somewhat contrary to the expectations of CK decrease in post-training measurement as the participants are getting accustomed to the increasing training load, no significant change in the level of muscle damage was observed, and even an increase of $23 \%$ in post-training CK was registered. Of course, a more demanding RS exercise, executed immediately prior to the posttraining measurement, was probably the main reason why there was no significant decrease in CK values. Additionally, the absence of significance in change can partially be explained by very high interindividual variability of CK (Russell, et al., 2015) which may influence the sensitivity of CK for longitudinal monitoring (Meister, der Fünten, \& Meyer, 2014). However, the fact that there was no significant increase in muscle damage post-training, even 
though the RS exercise done immediately prior to the post-training measuring point had $60 \%$ greater number of sprints and overall distance run, indicates a certain level of adaptation was developed to this type of training. It appears that this 6-week RST program is suitable for athletes of a similar fitness level as were the participants in this study, to maintain, or even alleviate, the level of muscle damage during the period of the increasing training load implementation, which is common for the preseason training in team sports. If the program had been too intensive and stressful for the participants, the acute increases in training load would probably have caused an accumulation of muscle damage. Such accumulation of CK throughout the intensified 6-week training period resulting in overreaching was already reported in rugby league players (Coutts, Reaburn, Piva, \& Rowsell, 2007). Obviously, the participants in our study were more on the path of getting accustomed than overreached to the constantly increasing training load.

The markers of OS increased following the training period and this increase in plasma $15-\mathrm{F}_{2 \mathrm{t}}-\mathrm{IsoP}$ was statistically significant. This was again contrary to our expectations as numerous studies showed a decrease of pro-oxidants following physical training in general (de Sousa, et al., 2017). Additionally, the results of just a few studies in which ARH following different types of HIIT were investigated actually reported the same. Bogdanis et al. (2013) reported a significant reduction of protein carbonyls (PC) and a non-significant decrease of thiobarbituric acid reactive substances (TBARS) following three weeks of sprint interval training consisting of four to six 30 -second all out sprints. A significant reduction of TBARS, but not of PC, was also reported following eight weeks of HIIT consisting of eighteen 1-minute intervals performed at 120 to $140 \%$ of $\mathrm{VO}_{2 \text { peak }}$ and separated by 2 -minute rest intervals performed at $65 \% \mathrm{VO}_{2 \text { peak }}$ in master runners (Vezzoli, et al., 2014). Finally, after only three HIIT sessions consisting of four 30 -second cycling sprints performed at $90 \%$ of maximal anaerobic power and separated by 4-minute active rest intervals, Fisher et al. (2011) reported a reduced acute response of TBARS following the session, indicating a reduction of oxidative stress as a result of even such a short training period. Generally, all HIIT formats investigated so far induced the same chronic adaptation after a training period, i.e. they led to the reduction of OS. Unfortunately, the only study that investigated ARH following an actual RST did not measure markers of OS, but only GPx, GR and SOD as markers of antioxidant capacity (Hellsten, et al., 1996), so no comparison with our study was possible. Contrary to the aforementioned studies, OS in this study was assessed through plasma and urinary $15-\mathrm{F}_{2 \mathrm{t}}$-IsoP levels as this marker had been proven to be stable in body fluids and, therefore, is considered the best marker of lipid peroxidation in vivo (Basu, 2004). Unfortunately, due to different blood analysis techniques utilized in other studies (Goods et al., 2016; Leeder et al., 2014), it was impossible to make any comparisons with the OS levels recorded in our study. Plasma and urinary $15-\mathrm{F}_{2 \mathrm{t}}-\mathrm{IsoP}$ levels were $75 \%$ and $25 \%$ greater at post-training, respectively, with the plasma $15-\mathrm{F}_{2 t}$-IsoP increase being statistically significant. Therefore, the main finding of our study was that following an intensive RST program significantly greater OS was obtained only in plasma marker, probably because the posttraining RS exercise had $60 \%$ greater workload in comparison to the pre-training one. The fact that urinary $15-\mathrm{F}_{2 t}-$ IsoP was not significantly greater at post-training may suggest that the training program did offer some kind of protection from OS. Namely, Knez et al. (2014) showed that constantly increased weekly training volume was responsible for the significantly increased OS following 30 $\mathrm{km}$ cycling time trial and this increase in OS was significantly associated with the increase in training volume $(r=0,71 ; \mathrm{p}<.001)$. The authors concluded that this was probably due to the inadequate recovery from training sessions as even resting OS significantly increased over the 8 -week training period. Additionally, acute OS response was shown to be intensity- and workload-dependent as significantly greater plasma lipid hydroperoxides concentrations were obtained following a low-volume high-intensity interval exercise than following a moderateintensity cycling bout (Wadley, Chen, Lip, Fisher, $\&$ Aldred, 2016). Therefore, greater levels of plasma and urinary $15-\mathrm{F}_{2 \mathrm{t}}$-IsoP obtained at post-training are probably the result of a combination of inadequate recovery, resulting from constantly increasing 6-week training volume, and a higher acute OS response due to the $60 \%$ greater workload of posttraining RS exercise. Assuming that a significant correlation between training volume and the posttraining $15-\mathrm{F}_{2 \mathrm{t}}$-IsoP levels exist (Knez, et al., 2014) and that the greater exercise workload elicits greater OS response (Wadley, et al., 2016), the fact that the increase in urinary $15-\mathrm{F}_{2 \mathrm{t}}$-IsoP level was much lower $(25 \%)$ than the increase of the total training volume from the first to the final week $(100 \%)$, and the increase of exercise workload (60\%) suggest that RST may actually cause attenuation of OS, conforming to the results of other studies analyzing exercise programs in general (de Sousa, et al., 2017).

Following RST, a decrease in GR, GPx and SOD was obtained, with only GR reaching statistical significance, thus indicating reduced antioxidant activity. On the other hand, serum UA showed a non-significant increase in the post-training measurement. Again, this was contrary to the findings of the meta-analysis indicating that physical training in general induced an increase in antioxidant param- 
eters (de Sousa, et al., 2017). This was also contrary to the results of the studies in which interval training programs were evaluated as significant increases in GPx, catalase (CAT) and total antioxidant capacity (TAC) were obtained following three weeks of HIIT program (Bogdanis, et al., 2013). On the other hand, no significant change in CAT, SOD and GPx was found after a HIIT program consisting of only three all-out sprint cycling sessions (Fisher, et al., 2011) as well as in resting TAC following four and eight weeks of HIIT (Vezzoli, et al., 2014). Also, no significant change in GPx, GR and SOD following six weeks of training was reported in the only study that investigated the effects of RST on muscle antioxidant enzymes (Hellsten, et al., 1996). However, in the same study, a significant increase in GPx and GR, but not in SOD was reported following one week of intensified RST, thus indicating that increased activity of muscle antioxidant enzymes was volume-dependent (Hellsten, et al., 1996). As no increase in muscle damage was observed, the authors suggested that this increase in post-training antioxidant activity was mainly due to an elevation in metabolically induced oxygen radical formation since cycling sprints were performed at the intensity corresponding to $300-400 \%$ of the intensity eliciting maximal oxygen uptake (Hellsten, et al., 1996). A volume-dependent significant increase in CAT following 8-week intensified cycling training was also observed by Knez et al. (2014). This increase in CAT was, however, a result of the upregulation of the antioxidant system in order to prevent excessive oxidative damage as significant increase in OS markers was also observed (Knez, et al., 2014). However, although chronic effects of exercise usually induce an increase in antioxidant activity, the acute reaction to high-intensity exercise might actually induce reduction in antioxidants, which may also be intensity-dependent. Namely, Farney et al. (2012) reported a significant decrease in CAT immediately after ten 15 -second sprints, while the decrease in CAT following 60 minutes of moderate-intensity aerobics was not statistically significant. The immediate non-significant reduction in CAT was also observed following the cycling RS exercise in the Peart et al. (2013) study as well. However, both studies actually reported an increase in antioxidants within one to two hours following this initial drop that occurred immediately post-exercise, thus exhibiting the underlying mechanism responsible for the chronic upregulation of antioxidant status (Farney, at al., 2012; Peart et al., 2013). Therefore, it seems that this significant reduction in GR, obtained immediately post-exercise following a training period in our study, was probably a result of a necessity for greater antioxidative counteract to a greater OS, caused by a $60 \%$ more stressful post-training RS exercise. The main ROS generating mechanism during RS exercise is probably ischemia-reperfusion process (Morales-Alamo \& Calbet, 2014) since intensive, anaerobic exercises are associated with a transient muscle hypoxia when oxygen supply cannot match energy demands (Vollaard, Shearman, \& Cooper, 2005). This is probably the reason why reduction was registered in SOD, GPx and GR, all involved in the ischemia-reperfusion process, while the nonsignificant increase was reported in UA, which is an end-product of purine metabolism (Finaud, et al., 2006) with a rather fast upregulating acute response to exercise via the activity of xanthine oxidase (Morales-Alamo \& Colbet, 2014). Therefore, this significant reduction of GR, probably stimulated with a significant increase of OS posttraining, might suggest that the participants were actually developing an antioxidant protection necessary to attenuate oxidative damage of their erythrocytes being imposed by an extremely stressful anaerobic exercise.

Although some studies suggest that muscle damage might be responsible for the increase in blood and muscle OS (Nikolaidis, et al., 2008), there were no significant associations between changes in $\mathrm{CK}$ and changes in any of the redox homeostasis markers found in our study. The absence of associations between the markers of muscle damage and OS was also found in other studies investigating acute responses to RS exercises (Bloomer, et al., 2006; Clifford, et al., 2016; Leeder, et al., 2014). In all these studies significant increase in CK was observed without the significant increase in OS markers, suggesting that muscle damage might have a limited role in inducing OS, at least during certain forms of RS exercises. High anaerobic fitness (Bloomer, et al., 2006), increased antioxidant enzyme activity after a prolonged intermittent-endurance training (Leeder, et al., 2014), and the difference in biochemical markers analyzed as well as the analytical techniques used (Clifford, et al., 2016) were all suggested as reasons for the absence of OS following a muscle damaging exercise. On the other hand, although the significant decrease in post-training CK as well as in the posttraining OS markers was observed following three weeks of sprint interval training (Bogdanis, et al., 2013), the authors did not statistically test associations between these variables, therefore, it remained unknown whether the post-training OS was really attenuated by the attenuation of muscle damage. Additionally, no connection of CK and antioxidant enzyme activities was found in the only running RST study investigating chronic effects (Hellsten, et al., 1996) so the authors concluded that the increase in GPx and GR post-training was mostly due to the metabolically induced oxygen radical formation. It appears that the level of muscle damage needs to be rather great, like after a downhill running, to become a major contributor to the increase in OS 
as after a muscle-damaging exercise, OS is mostly achieved through the oxidation of neutrophils and other phagocytic cells engaged in tissue reparation (Nikolaidis, et al., 2008). Although the exercise mode was running and, therefore, the level of CK was high in this study, the pre- to post-training change in CK was obviously not that great to present a significant impact on the significant increase and decrease of post-training OS and GR, respectively. This was probably because even the pre-training RS exercise was highly muscle damaging and posttraining RS exercise could not elicit any further increase in CK. Additionally, the chosen assessment time points at which biological material was sampled might also be contributors to the blurring of any possible associations between them. Namely, peak CK is usually obtained one or even two days post-exercise, while the OS markers peak within several hours post-exercise (Michailidis, et al., 2007). Anyway, it seems that the adaptations in redox homeostasis were more altered through other OS mechanisms, such as the ischemia-reperfusion process and lactate production (Finaud, et al., 2006), as RS exercises were of high anaerobic nature (Buchheit \& Laursen, 2013b).
The limitation of the study was that blood and urine samples were not taken before and at several time points after the first and the penultimate RS training session; consequently, an acute reaction to RS exercises could not be determined. Also, it was impossible to compare $15-\mathrm{F}_{2 \mathrm{t}}$-IsoP results between the studies due to the lack of method standardization.

In conclusion, we showed for the first time that RST may stimulate the balance in redox homeostasis. Significant increase in OS coupled with a significant reduction in GR at post-training are probably the result of a more stressful post-training RS exercise and, therefore, may suggest that RTS actually leads to the reduction in OS and may provide antioxidant protection. The level of muscle damage following RS exercise was not changed after the RST program and showed not to be associated with changes in markers representing ARH. Our findings suggest that healthy trained individuals involved in RS exercises should not be concerned about the increased oxidative stress because such a condition does not seem to occur in this type of training intervention.

\section{References}

Basu, S. (2004). Isoprostanes: Novel bioactive products of lipid peroxidation. Free Radical Research, 38(2), 105-122.

Batacan, R.B., Duncan, M.J., Dalbo, V.J., Tucker, P.S., \& Fenning, A.S. (2017). Effects of high-intensity interval training on cardiometabolic health: A systematic review and meta-analysis of intervention studies. British Journal of Sports Medicine, 51(6), 494-503.

Bishop, D., Girard, O., \& Mendez-Villanueva, A. (2011). Repeated-sprint ability - Part II. Recommendations for training. Sports Medicine, 41(9), 741-756.

Bloomer, R.J., Falvo, M.J., Fry, A.C., Schilling, B.K., Smith, W.A., \& Moore, C.A. (2006). Oxidative stress response in trained men following repeated squats or sprints. Medicine and Science in Sports and Exercise, 38(8), 1436-1442.

Bogdanis, G.C., Stavrinou, P., Fatouros, I.G., Philippou, A., Chatzinikolaou, A., Draganidis, D., Ermidis, G., \& Maridaki, M. (2013). Short-term high-intensity interval exercise training attenuates oxidative stress responses and improves antioxidant status in healthy humans. Food and Chemical Toxicology, 61, 171-177.

Bok, D., \& Jukić, I. (2019). Muscle damage during a soccer World Cup preparatory and competition period. International Journal of Sports Physiology and Performance, ahead of print. doi: https://doi.org/10.1123/ ijspp.2019-0084

Bok, D., Jukić, I., Milanović, D., \& Gregov, C. (2015). Improving repeated sprint ability: Repeated sprint vs shuttle repeated sprint training. Medicine and Science in Sports and Exercise, 47(5, Suppl. 1), 543.

Bouzid, M.A., Filaire, E., McCall, A., \& Fabre, C. (2015). Radical oxygen species, exercise and aging: An update. Sports Medicine, 45, 1245-1261.

Buchheit, M., \& Laursen, P.B. (2013a). High-intensity interval training, solutions to the programming puzzle. Part 1: Cardiopulmonary emphasis. Sports Medicine, 43(5), 313-338.

Buchheit, M., \& Laursen, P.B. (2013b). High-intensity interval training, solutions to the programming puzzle. Part 2: Anaerobic energy, neuromuscular load and practical application. Sports Medicine, 43(10), 927-954.

Clifford, T., Berntzen, B., Davison, G.W., West, D.J., Howatson, G., \& Stevenson, E.J. (2016). Effects of beetroot juice on recovery of muscle function and performance between bouts of repeated sprint exercise. Nutrients, 8(8), 506. doi: 10.3390/nu8080506. 
Coutts, A.J., Reaburn, P., Piva, T.J., \& Rowsell, G.J. (2007). Monitoring for overreaching in rugby league players. European Journal of Applied Physiology, 99(3), 313-324.

de Sousa, C.V., Sales, M.M., Rosa, T.S., Lewis, J.E., de Andrade, R.V., \& Simões, H.G. (2017). The antioxidant effect of exercise: A systematic review and meta-analysis. Sports Medicine, 42(2), 277-293.

Douglas, J., Pearson, S., Ross, A., \& McGuigan, M. (2016). Eccentric exercise: Physiological characteristics and acute responses. Sports Medicine, 47(4), 663-675.

Escobar, M., Oliveira, M.W.S., Behr, G.A., Zanotto-Filho, A., Ilha, L., Dos Santos Cunha, G., de Oliveira, A.R., \& Moreira, J.C.F. (2009). Oxidative stress in young football (soccer) players in intermittent high intensity exercise protocol. Journal of Exercise Physiology Online, 12(5), 1-10.

Farney, T.M., McCarthy, C.G., Canale, R.E., Schilling, B.K., Whitehead, P.N., \& Bloomer, R.J. (2012). Absence of blood oxidative stress in trained men after strenuous exercise. Medicine and Science in Sports and Exercise, 44(10), 1855-1863.

Finaud, J., Lac, G., \& Filaire, E. (2006). Oxidative stress: Relationship with exercise and training. Sports Medicine, 36(4), 327-358.

Fisher, G., Schwartz, D.D., Quindry, J., Barberio, M.D., Foster, E.B., Jones, K.W., \& Pascoe, D.D. (2011). Lymphocyte enzymatic antioxidant responses to oxidative stress following high-intensity interval exercise. Journal of Applied Physiology, 110(3), 730-737.

Garber, C.E., Blissmer, B., Deschenes, M.R., Franklin, B.A., Lamonte, M.J., Lee, I.M., Nieman, D.C., \& Swain, D.P. (2011). Quantity and quality of exercise for developing and maintaining cardiorespiratory, musculoskeletal, and neuromotor fitness in apparently healthy adults: Guidance for prescribing exercise. Medicine and Science in Sports and Exercise, 43(7), 1334-1359.

Gibala, M. (2007). High-intensity interval training: A time-efficient strategy for health promotion. Current Sports Medicine Reports, 6(4), 211-213.

Goods, P.S.R., Dawson, B., Landers, G.J., Gore, C.J., Croft, K., \& Peeling, P. (2016). Effect of repeatedsprint training in hypoxia on post-exercise interleukin-6 and F2-isoprostanes. European Journal of Sport Science, 16(8), 1047-1054.

Hellsten, Y., Apple, F.S., \& Sjödin, B. (1996). Effect of sprint cycle training on activities of antioxidant enzymes in human skeletal muscle. Journal of Applied Physiology, 81(4), 1484-1487.

Howatson, G., \& Milak, A. (2009). Exercise-induced muscle damage following a bout of sport specific repeated sprints. Journal of Strength and Conditioning Research, 23(8), 2419-2424.

Jówko, E., Długołęcka, B., Makaruk, B., \& Cieśliński, I. (2015). The effect of green tea extract supplementation on exercise-induced oxidative stress parameters in male sprinters. European Journal of Nutrition, 54(5), 783-791.

Knez, W.L., Jenkins, D.G., \& Coombes, J.S. (2014). The effect of an increased training volume on oxidative stress. International Journal of Sports Medicine, 35(1), 8-13.

Leeder, J.D.C., van Someren, K.A., Gaze, D., Jewell, A., Deshmukh, N.I.K., Shah, I., Barker, J., \& Howatson, G. (2014). Recovery and adaptation from repeated intermittent-sprint exercise. International Journal of Sports Physiology and Performance, 9(3), 489-496.

Lewis, N.A., Howatson, G., Morton, K., Hill, J., \& Pedlar, C.R. (2015). Alterations in redox homeostasis in the elite endurance athlete. Sports Medicine, 45(3), 379-409.

Margonis, K., Fatouros. I.G., Jamurtas, A.Z., Nikolaidis, M.G., Douroudos, I., Chatzinikolaou, A., Mitrakou, A., Mastorakos, G., Papassotiriou, I., Taxildaris, K., \& Kouretas, D. (2007). Oxidative stress biomarkers responses to physical overtraining: Implications for diagnosis. Free Radical Biology and Medicine, 43(6), 901-910.

Meister, S., der Fünten, K., \& Meyer, T. (2014). Repeated monitoring of blood parameters for evaluating strain and overload in elite football players: Is it justified? Journal of Sports Sciences, 32(13), 1328-1331.

Michialidis, Y., Jamurtas, A.Z., Nikolaidis, M.G., Fatouros, I.G., Koutedakis, Y., Papassotiriou, I., Kouretas, D. (2007). Sampling time is crucial for measurement of aerobic exercise-induced oxidative stress. Medicine and Science in Sports and Exercise, 39(7), 1107-1113.

Morales-Alamo, D., \& Calbet, J.A.L. (2014). Free radicals and sprint exercise in humans. Free Radical Research, 48(1), 30-42.

Nikolac, N., Šupak-Smolčić, V., Šimundić, A.M., \& Ćelap, I. (2013). Croatian Society of Medical Biochemistry and Laboratory Medicine: National recommendations for venous blood sampling. Biochemia Medica, 23(3), 242-254.

Nikolaidis, M.G., Jamurtas, A.Z., Paschalis, V., Fatouros, I.G., Koutedakis, Y., \& Kouretas, D. (2008). The effect of muscle-damaging exercise on blood and skeletal muscle oxidative stress: Magnitude and timecourse considerations. Sports Medicine, 38(7), 579-606. 
Peart, D.J., Kirk, R.J., Hillman, A.R., Madden, L.A., Siegler, J.C., \& Vince, R.V. (2013). The physiological stress response to high-intensity sprint exercise following the ingestion of sodium bicarbonate. European Journal of Applied Physiology, 113(1), 127-134.

Russell, M., Northeast, J., Atkinson, G., Shearer, D.A., Sparkes, W., Cook, C.J., \& Kilduff, L.P. (2015). Between-match variability of peak power output and creatine kinase responses to soccer match-play. Journal of Strength and Conditioning Research, 29(8), 2079-2085.

Silva, J.R., Rumpf, M.C., Hertzog, M., Castagna, C., Farooq, A., Girard, O., \& Hader, K. (2018). Acute and residual soccer match-related fatigue: A systematic review and meta-analysis. Sports Medicine, 48(3), 539-583.

Taylor, J., Macpherson, T., Spears, I., \& Weston, M. (2015). The effects of repeated-sprint training on fieldbased fitness measures: A meta-analysis of controlled and non-controlled trials. Sports Medicine, 45(6), 881-891.

Vezzoli, A., Pugliese, L., Marzorati, M., Serpiello, F.R., La Torre, A., \& Porcelli, S. (2014). Time-course changes of oxidative stress response to high-intensity discontinuous training versus moderate-intensity continuous training in master runners. PLoS One, 9(1), e87506

Vollaard, N.B.J., Shearman, J.P., \& Cooper, C.E. (2005). Exercise induced oxidative stress: Myths, realities and physiological relevance. Sports Medicine, 35(12), 1045-1062.

Wadley, A.J., Chen, Y.W., Lip, G.Y.H., Fisher, J.P., \& Aldred, S. (2016). Low volume-high intensity interval exercise elicits antioxidant and anti-inflammatory effects in humans. Journal of Sports Sciences, 34(1), 1-9.

Weston, M., Taylor, K.L., Batterham, A.M., \& Hopkins, W.G. (2014). Effects of low-volume high-intensity interval training (HIIT) on fitness in adults: A meta-analysis of controlled and non-controlled trials. Sports Medicine, 44(7), 1005-1017.

Weston, K.S., Wisløff, U., \& Coombes, J.S. (2014). High-intensity interval training in patients with lifestyleinduced cardiometabolic disease: A systematic review and meta-analysis. British Journal of Sports Medicine, 48(16), 1227-1234.

Woolley, B.P., Jakeman, J.R., \& Faulkner, J.A. (2014). Multiple sprint exercise with a short deceleration induces muscle damage and performance impairment in young, physically active males. Journal of Athletic Enhancement, 3(2). doi: http://dx.doi.org/10.4172/2324-9080.1000144

Submitted: November 13, 2019

Accepted: January 21, 2020

Published Online First: March 9, 2020

Correspondence to:

Domagoj Marijančević, M.D.

European Specialist in Laboratory Medicine

(EuSpLM)

Endocrinology Laboratory

Department of Oncology and Nuclear Medicine

Sestre Milosrdnice University Hospital Center

Vinogradska 29, 10000 Zagreb, Croatia

Phone: +385989480437 ,

E-mail: domagoj.marijancevic@kbcsm.hr 